\title{
Maximum Energy Welfare Routing in Wireless Sensor Networks
}

\author{
Changsoo $\mathrm{Ok}^{1}$, Prasenjit Mitra ${ }^{2}$, Seokcheon Lee ${ }^{3}$, and Soundar Kumara ${ }^{1}$ \\ ${ }^{1}$ The Department of Industrial Engineering, The Pennsylvania State University, \\ University Park, PA 16802, USA \\ \{cuo108, skumara\}@psu .edu \\ ${ }^{2}$ College of Information Sciences and Technology, The Pennsylvania State University, \\ University Park, PA 16802, USA \\ pmitra@ist.psu.edu \\ 3 The School of Industrial Engineering, Purdue University, \\ West Lafayette, IN 47907-20232, USA \\ lee46@purdue.edu
}

\begin{abstract}
Most routing algorithms for sensor networks focus on finding energy efficient paths to prolong the lifetime of sensor networks. As a result, the sensors on the efficient paths are depleted quickly, and consequently the sensor networks become incapable of monitoring events from some parts of their target areas. In many sensor network applications, the events have uncertainties in positions and generation patterns. Therefore, routing algorithms should be designed to consider not only energy efficiency, but also the amount of energy left in each sensor to avoid sensors running out of power early. This paper introduces a new metric, called Energy-Welfare, devised to consider average and balance of sensors' remaining energies simultaneously. Using this metric, we design the Maximum Energy Welfare Routing algorithm, which achieves energy efficiency and energy balance of sensor networks simultaneously. Moreover, we demonstrate the effectiveness of the proposed routing algorithm by comparing with three existing routing algorithms.
\end{abstract}

Keywords: Sensor Network, Distributed algorithm, Energy aware routing, Energy Welfare, Social welfare, Energy balance.

\section{Introduction}

Sensor networks report predetermined events or transmit sensed data to the base station for further analysis $[1,2]$. The sensors contain a fixed amount of stored power and the process of sending data consumes some of that stored power. It is desirable that the sensors run as long as possible. In this work, we propose a routing algorithm that attempts to route messages efficiently so as to maximize the life of a sensor network. Consequently, the design of the routing algorithm for sensor networks should also incorporate the following factors [2].

- Due to sensors' limited power, the routing algorithm should have a design to allow finding efficient paths to prolong the lifetime of the sensor network. 
- However, it is inevitable for most energy efficient routing algorithms to drive some sensors, which are close to the base station or on energy efficient paths, drained power quickly. As a result, the sensor networks become unable to detect events from regions where all sensors are nonfunctioning. Thus, in the sensor networks, data traffic should be dispersed and distributed over the whole network to extend its lifetime.

- Although most existing routing algorithms assume that events are generated uniformly at each sensor, events could occur randomly [3], uniformly [4] over the target area, or repeatedly [5] at a specific part of the target area. Event patterns can change from one type to another over time. Therefore, the routing algorithm should be sufficiently robust for diverse event generation functions. This problem can be addressed by routing so as to utilize the energy uniformly over the entire sensor network.

- In addition, a sensor network can consist of a large number of nodes for which a central control architecture does not apply. Therefore, the routing algorithm should adopt a local decision making scheme.

Although the literature includes several routing algorithms, such as direct communication approach, hierarchical routing methods [6, 7], self-organized routing algorithm [4], and the other routing algorithms [8], little evidence exists for effectiveness and efficiency of these algorithms with respect to the considerations mentioned earlier.
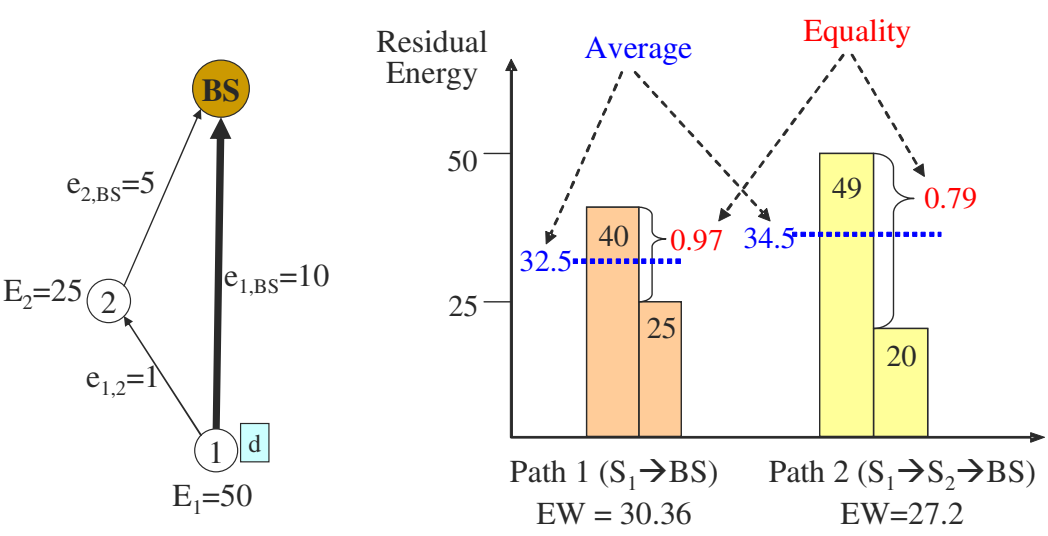

Fig. 1. An explanatory example for Energy Welfare Routing algorithm: Node 1 routes data to a path to maximize an Energy Welfare (AveragexEquality) of sensor 1 and 2

We assume that the neighbors only get the information about the energy left in each neighbor and the energy required to transmit to the base station from that neighbor periodically. Individual sensors forward messages to neighbors that they think are on the "best" route to the base station. The determination of the optimal route is difficult because the individual sensors do not have the information about the 
dynamic topology of the entire network and the dynamic energy balances of each node on the network. This study proposes a new heuristic metric, called Energy Welfare, to achieve the efficiency and balance of energies of sensors simultaneously. Based on this metric, we propose a localized routing algorithm, the Maximum Energy Welfare (MaxEW) routing, to accomplish the two objectives. Figure 1 gives a simple example of the MaxEW routing. $E_{i}$ and $e_{i j}$ represent the residual energy of sensor $i$ and energy required to transmit from node $i$ to node $j$ respectively. Sensor 1 has two paths to reach to the base station. Path 2 is more energy efficient than Path 1 . However, if sensor 1 keeps using path 2, sensor 2 will run out its power while sensor 1 has sufficient energy. In MaxEW, sensors can avoid the traffic concentration to a sensor by using a metric, Energy Welfare (Average $\times$ Equality), as a decision criterion. The Equality is in inverse proportion to the difference between energy levels of sensor 1 and 2. Based on the metric, sensor 1 chooses path 1 which causes higher energy welfare.

The rest of this paper has the following organization: Section 2 presents the details of the considered sensor network. Section 3 defines energy equality and the welfare of sensor network as new metrics. After describing the details of the maximum energywelfare routing algorithms in Section 4, Section 5 presents extensive simulation results, and Section 6 details conclusions.

\section{Sensor Network Model}

With $n$ homogenous sensors randomly and uniformly distributed over a target area, every sensed data must be sent to the base station. Each sensor has limited battery power. Sensors can control their respective transmission power for minimal consumption to transmit to a destination $[6,7]$ and they have discrete adjustable transmission power levels [9-12]. This ability is necessary to allow the routing algorithm to maximize sensor networks' operational times. Therefore, sensors can send data to either a neighbor or the base station directly, according to their routing policies $[4,6,7]$. The details of the problem are:

\subsection{Energy Consumption Model}

Each sensor uses a fixed transmission power for communicating with its neighboring sensors while each sensor transmits data to the base station. The neighboring distance is defined as the maximal reachable distance with the fixed transmission power for neighboring sensors. For a given sensor the sensors within its neighboring distance are its "neighboring sensors" or "neighbors". In this scheme, each node can be aware of the current energy level of its neighbors or energy required to transmit from its neighboring nodes to the base station by anticipating and/or eavesdropping data from the neighbors.

Generally, sensors use their energy when they sense, receive and transmit data. However, the amount of energy consumption for sensing is unaffected by the routing algorithm and only a small difference exists between the power consumption of idle and receiving modes [13]. Therefore, consideration is only energy consumption by 
transmission in the design of the routing algorithms to maximize the lifetime of the sensor network. By normalization of the radio characteristic constant and the size of sensed data [4], the energy consumption model is simplified to $E=d^{2}$, where $E$ and $d$ are the required energy and the transmission distance respectively.

\subsection{The Lifetime of Sensor Network}

Validating the effectiveness of the proposed MaxEW routing algorithm uses the lifetime of sensor network as the performance measure $[4,6,8,11]$. The definition of lifetime of a sensor network is the time or number of rounds that occur until the first node or a portion of nodes become incapable, due to energy depletion, of sending data to the base station directly or indirectly via its neighbors $[4,6,8,11$, 14]. The portion (number of depleted nodes) can vary depending on the context of the sensor networks. In this paper, the lifetime of a sensor network is the number of rounds until the first $\left(\mathrm{L}_{1}\right), 10 \%\left(\mathrm{~L}_{10}\right)$, or $20 \%\left(\mathrm{~L}_{20}\right)$ node(s) expend all their power $[8,11]$.

\subsection{Event Generation Functions}

For evaluation purposes, many previous studies of routing algorithms assumed that all sensors have uniform data or event generation rates [4, 6, 7]. In infrastructure monitoring applications, each sensor performs a sensing task every time, $T$, and has a homogeneous event generation function. However, in many sensor network applications, this assumption becomes unrealistic. In a monitoring application for the migration of a herd of animals, the animals might move along a path in the target area repeatedly [5]. While, in the case of forest fire detection, events can occur rarely and randomly over the target area [3]. Furthermore, some event generation functions can be a combination of uniform, random, and repeated types. Therefore, more reasonable is the consideration of several event types for evaluation of routing algorithms. The results section demonstrates that our algorithm is robust for the different types of event generation functions.

\section{Energy Equality and Welfare}

To keep detecting events at an unknown position in a target area for as long as possible, routing algorithms should have a design to enable finding efficient paths, and, at the same time, prevent a particular set of sensors from being depleted early by a concentration of data traffic. In other words, the routing algorithms must achieve an energy balance for sensor networks while guaranteeing that sensors use their energies efficiently. Designing the routing algorithm first required a new measure for considering energy balance of sensor networks, as well as energy efficiency of routing algorithms. For this purpose, two definitions of Energy-Equality (EE) and EnergyWelfare (EW) apply. 


\subsection{Energy-Equality (EE)}

To measure how well energy-balanced a sensor network is, we define the EnergyEquality (EE) of a given sensor network is Equation (1) and (2):

$$
\begin{aligned}
& E E(t, \mathcal{E})=1-I_{N}(t, \mathcal{E}) . \\
& I_{N}(t, \mathcal{\varepsilon})=1-\left[\frac{1}{n} \sum_{i \in N}\left(\frac{E_{i}(t)}{E(t)}\right)^{1-\varepsilon}\right]^{\frac{1}{1-\varepsilon}} .
\end{aligned}
$$

$I_{N}(t, \mathcal{E})$ is the inequality(explained below) of the sensor's residual batteries at time, $t . N$ and $\varepsilon$ are the set of nodes in the sensor network and the inequality aversion parameter. $n$ and $E_{i}(t)$ are the number of sensors and the remaining energy of sensor, $i$, at time, $t$, respectively. Basically, the derivation of the energy inequality index, $I_{N}(t, \varepsilon)$, is from the Atkinson inequality index [15]. Social scientists use the index to measure the inequality among entities with respect to their income. The aversion parameter $\mathcal{E}$ reflects the strength of society's penalty for inequality, and can take values ranging from zero to infinity. When $\varepsilon$ equals zero, no penalty for inequality accrues. As $\varepsilon$ rises, society penalizes inequality more. The values of $\mathcal{E}$ that are typically used include, 1.5 and 2.5 [16, 17]. This aversion parameter provides a flexibility to apply this metric to diverse sensor network applications.

\subsection{Energy-Welfare (EW)}

A drawback exists for only considering energy balance of a sensor network. If a routing algorithm only pursues the energy balance without considering energy efficiency, sensors' residual energies might converge to a lower value. That is, sensors possibly use their energy in an inefficient way to achieve the energy-balance of the sensor network. Therefore, Energy-Welfare $(E W)$ is the consideration of energy efficiency and energy balance of sensor networks simultaneously. $E W$ is a simple form of weighting the average of sensors' residual energies by $E E$. We can calculate $E W$ using equation (3).

$$
E W(t)=\overline{E(t)} \times E E(t, \varepsilon)
$$

The equation for $E W$ has the same form as Atkinson welfare function [18]. The $E W$ is high where the average and equality of sensors' remaining battery power are both high. A low average, or $E E$, leads to a low $E W$. Therefore, the $E W$ is an appropriate metric to design a routing algorithm that improves sensor networks with energy efficiency and energy balance perspectives. Additionally, since a sensor 
network having a high $E W$, can monitor unknown position events for a long time, the $E W$ has consideration as the preparedness of sensor networks for upcoming events.

\section{Maximum Energy Welfare (MaxEW) Routing}

In this paper, we assume that each sensor uses a fixed transmission power for communicating with its neighboring nodes. To send a message from a sensor to the base station the total transmission power required is minimized if the sensor communicates directly with the base station. The sensors are aware of the minimum transmission power required to send a message to the base station and the current battery level of its neighbors.

The basic idea of the proposed routing algorithm is simply to use a path which maximizes the $E W$ of the sensors. When a node $i$ needs to send data to the base station, it can transmit data to the base station directly or route the data to one of its neighbors $\left(N_{i}\right)$. For evaluating these alternatives, node $i$ calculates the $E W$ of a local society which consists of its neighbor and itself for each alterative. That is, the node can anticipate the residual energies of its neighboring nodes and itself when the data is routed according to its decision as in (4).

$$
\left\{E_{j}(t): j \in N_{i}+\{i\}\right\} \stackrel{D_{i}(t)}{\rightarrow}\left\{E_{j}(t+1): j \in N_{i}+\{i\}\right\}
$$

Also, this node can calculate the $E W_{i j}$ of these expected residual energies, $E_{j}(t+1)$, for each decision, $D_{i}(t)$, by (5). By comparing these expected $E W \mathrm{~s}$, the node routes data to the path allowing the maximum $E W$ of the local society, $N_{i}+\{i\}$.

$$
E W_{i j}(t+1)=\overline{E(t+1)}\left(1-I_{N(\varepsilon)}(t+1)\right) .
$$

Through this decision making scheme, MaxEW tries to maximize the energy welfare of the entire sensor network. In MaxEW, each sensor keeps a small size routing table only for its neighboring nodes. The routing table contains node identification number, minimum transmission power to the base station, and available energy, for each neighbor node. The details of the algorithm are:

Initialize routing table. During the setup period, each sensor finds its minimum transmission power to the base station. Then, each sensor broadcasts a setup message to neighboring nodes with a pre-set transmission power. This setup message includes node ID., minimum transmission power to the base station, and available energy. Every node receiving this broadcast message registers the transmitting node as one of its neighbors. Since all nodes have an identical neighbor distance, two nodes within the neighbor distance are neighbors to each other. After the setup period, all sensors initialize their routing tables. 


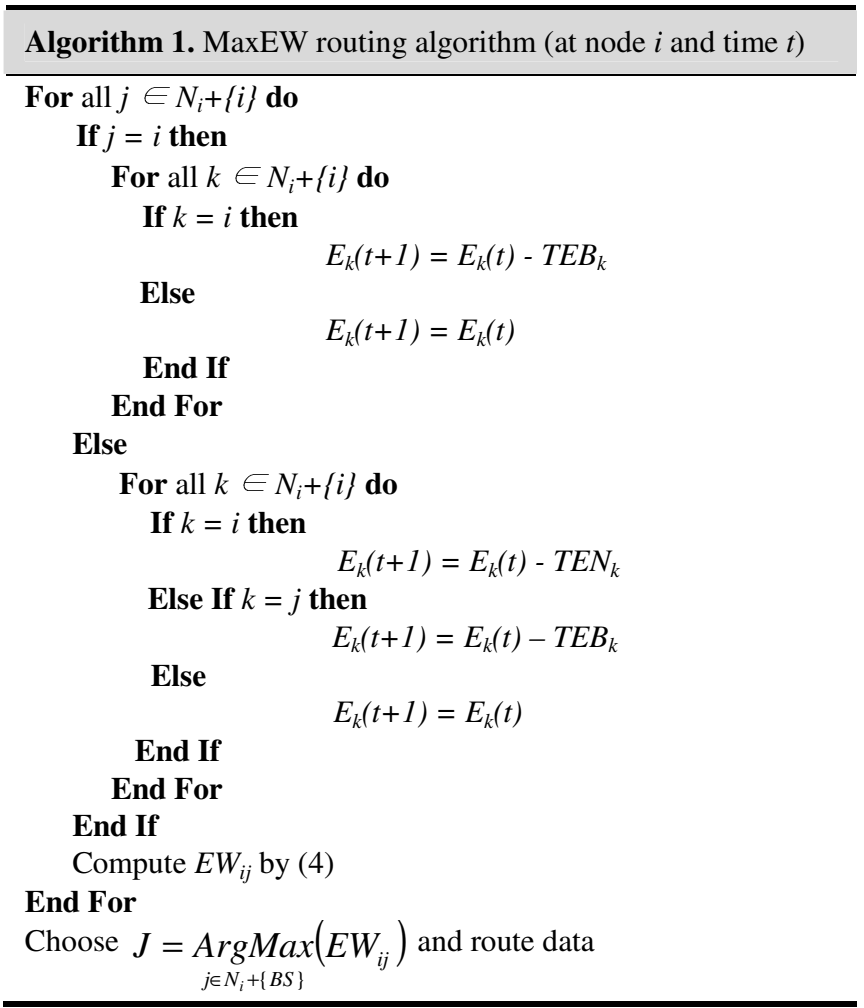

Update routing table. A change of a neighbor's energy level should be reflected in the routing table. When a sensor transmits data, all of its neighbors receive this data and get the current battery level of the transmitting sensor. As a result, whenever a sensor's battery level changes, all routing tables including the corresponding sensor information are updated.

Localized routing decision. Based on their routing tables, every node makes a local routing decision. Algorithm 1 gives a high-level description of the MaxEW algorithm of node $i$, at time, $t . T E B_{k}$ and $T E N_{k}$ are the required transmission energies from node, $k$, to the base station and neighboring nodes, respectively. Also, $E W_{i j}$ is the expected energy welfare of $N_{i}+\{i\}$ when node $i$ routes data to node, $j$. Based on this algorithm, node $i$ selects $J$ as the best candidate for transmitting data to the base station without considering whether $J$ sends data directly to the base station or not. If the selection is that node $i$ itself is the best node, it sends the data to the base station, finishing the routing process. Otherwise, the data is routed to node $J$ and $J$ performs the same process. This process continues until the base station receives the data. This localized decision making process results in a monotonic increase of EW because the best candidate can have a better indirect path than direct transmission. 


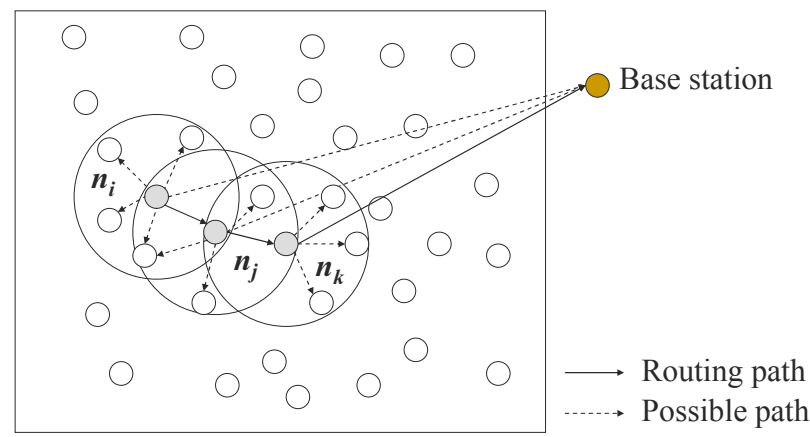

Fig. 2. An example routing path: $n_{i}$ sends data to $n_{j}, n_{j}$ to $n_{k}$, then $n_{k}$ sends to the base station directly

Fig. 2 shows how the MaxEW algorithm operates over a sensor network. For a given data, $n_{i}$ chooses $n_{j}$ among several possible routes. After the data passes to $n_{j}$, energy level of $n_{i}$ changes and the routing table of $n_{j}$ also changes. $n_{j}$ performs the same process sequentially. In the figure, $n_{k}$ sends data to the base station directly because the transmission of $n_{k}$, itself results in the maximum EW of $n_{k}$ 's local society compared to other neighbors. MaxEW guarantees elimination of loops in any routing path. In MaxEW, a sensor routes data to a neighbor only if the neighbor incurs more energy welfare than the sensor itself. As this routing mechanism continues, the expected energy welfare of the original node is apparently greater than that of the next down-stream node. Therefore, MaxEW always assures finding a routing path to the base station without loops.

\section{Experimental Results}

In this section, several experimental results validate the effectiveness of the MaxEW algorithm. The comparison of the algorithm is with three other algorithms discussed in [4, 7]: Direct Communication (DC), Minimum Transmission Energy (MTE), and Self-Organized routing (SOR). In DC, every sensor simply transmits data directly to the base station without considering any energy-efficient indirect path. MTE and SOR consider indirect routing to save sensor power but make routing decisions based on energy efficiency only. The MaxEW algorithm tries to achieve an energy balance of the network by maximizing the EW of a local society in a decentralized manner. Experimental results show that this approach is valid for extending the lifetime of sensor networks and robust for different event.

The experiments use a sensor network in which 100 nodes have random and uniform deployment in a $100 m \times 100 m$ square area with the base station located at $(50$, 150)(see Fig. 3). In the sensor network, sensors have an initial battery level of 250,000. The initial energy levels are established by determining the amount of energy needed for the farthest node to transmit data to the base station 100 times with DC and also used in [4]. To discuss the effect of different event generation types on the lifetime of 


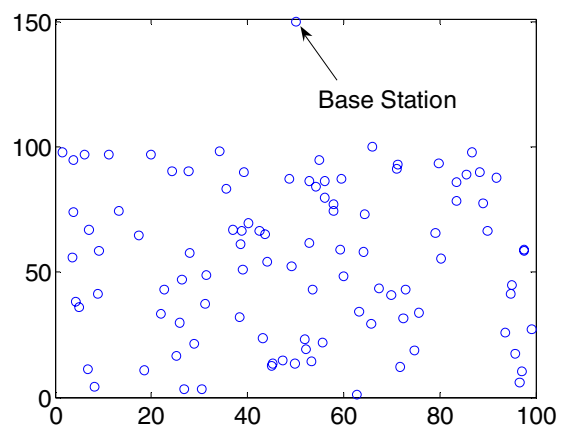

Fig. 3. The configurations of the experimental sensor networks: 100 nodes, randomly and uniformly deployed in $100 \mathrm{~m} \times 100 \mathrm{~m}$ square area, have the base station located at $(50,150)[6,7]$

a sensor network, performed simulations uses uniform, random, and repeat event generation functions. In the case of the random distribution, $25 \%$ of sensors have events randomly occurring in each round. While, for the repeat events, the assumption is that sensors from $(0,0)$ to $(50,50)$ incur repeated events. Because a sensor network is generated randomly, 100 repeated experiments for each condition provides an average of the results. Lastly, neighbor distance of sensors (for MTE and MaxEW) and the aversion parameter $\mathcal{E}$ (for MaxEW) have settings of $15 \mathrm{~m}$ and 2.5 respectively.

Table 1. Lifetime $\left(\mathrm{L}_{1}, \mathrm{~L}_{10}, \mathrm{~L}_{20}\right)$ for Direct, MTE, SOR, and MaxEW with Uniform, Random, and Repeat Events

\begin{tabular}{lrrrrrrrrr}
\hline & \multicolumn{3}{c}{ Uniform } & \multicolumn{3}{c}{ Random } & \multicolumn{3}{c}{ Repeat } \\
\hline & \multicolumn{1}{c}{$\mathrm{L}_{1}$} & \multicolumn{1}{c}{$\mathrm{L}_{10}$} & \multicolumn{1}{c}{$\mathrm{L}_{20}$} & \multicolumn{1}{c}{$\mathrm{L}_{1}$} & \multicolumn{1}{c}{$\mathrm{L}_{10}$} & $\mathrm{~L}_{20}$ & $\mathrm{~L}_{1}$ & $\mathrm{~L}_{10}$ & $\mathrm{~L}_{20}$ \\
\hline Direct & 105.3 & 123.4 & 142.3 & 492.7 & 618.1 & 717.2 & 107.8 & 145.8 & 193.3 \\
\hline MTE & 14.4 & 67.1 & 115.5 & 74.6 & 337.4 & 581.1 & 30.0 & 223.7 & 415.6 \\
\hline SOR & 28.7 & 145.9 & 109.9 & 111.3 & 562.9 & 771.4 & 154.1 & 316.9 & 418.2 \\
\hline MaxEW & 202.5 & 258.3 & 268.9 & 1012.7 & 1239.9 & 1346.7 & 685.6 & 970.6 & 1020.6 \\
\hline
\end{tabular}

Table 1 gives the result for the lifetime of sensor network $\left(\mathrm{L}_{1}, \mathrm{~L}_{10}, \mathrm{~L}_{20}\right)$ for Direct Communication, MTE, SOR, and MaxEW algorithms with three different event generation types. As shown in Table1, MaxEW shows a dominant performance compared with Direct, MTE and SOR over the time. Especially, in the case of $\mathrm{L}_{1}$, MaxEW gives approximately two to eight times better performance than the others.

Fig. 4 shows how well MaxEW achieves the energy balance of sensors over the network. As discussed in [7], in the Direct (Fig. 4(a)), SOR (Fig. 4(c)) and MTE (Fig. 4(b)) routing scheme sensors far away and close to the base station ran out their energies around 150 round. While, in MaxEW, all sensors remain live and even have sufficient energy for responding upcoming events (Fig. 4(d)). Also notable is that Direct, MTE, and SOR missed some events during the first 150 rounds. However, MaxEW guaranteed that all data is transmitted to the base station for the same period. 


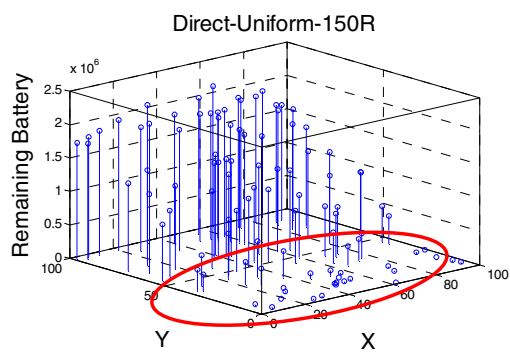

(a)

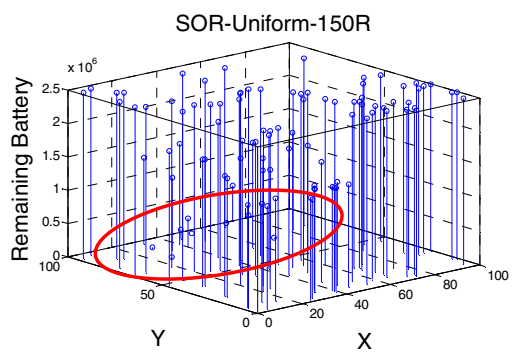

(c)

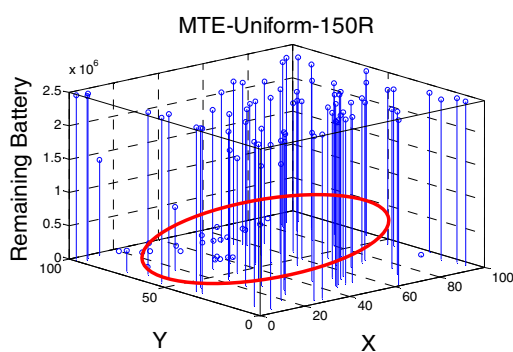

(b)

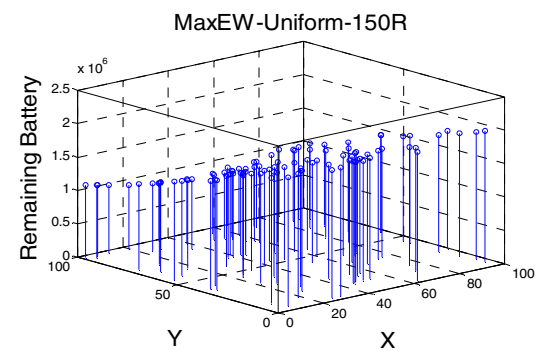

(d)

Fig. 4. The remaining energy distributions of sensors with uniform events at the 150 Round for DC, DTE, SOR, and MaxEW

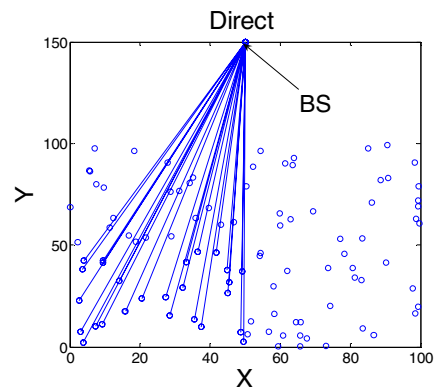

(a)

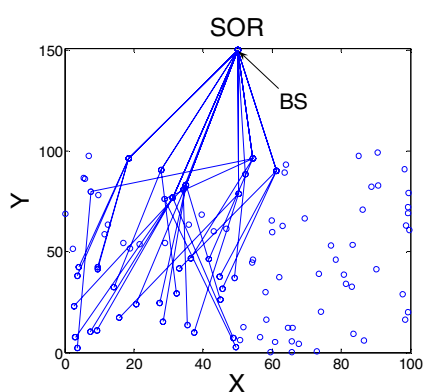

(c)

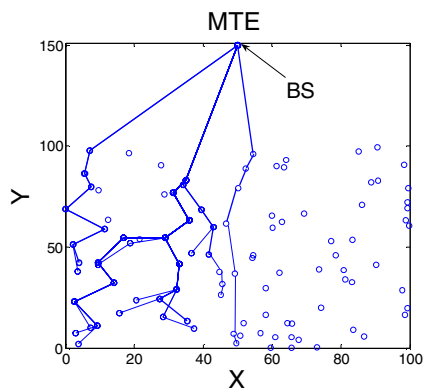

(b)

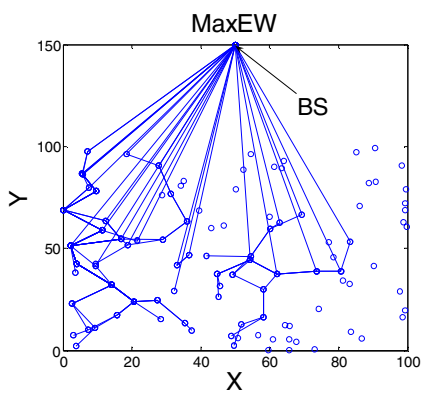

(d)

Fig. 5. Routing paths by Direct, MTE, SOR, and MaxEW with repeat events on the region from $(0,0)$ to $(50,50)$ 
Fig. 5 shows the routing paths for four algorithms with repeated events in the regions from $(0,0)$ to $(50,50)$. In the case of Direct, MTE, and SOR, data traffic concentrates in specific sensors which have location in the region or on the efficient path. On the other hand, MaxEW tries to dissipate energy usage over the whole network to achieve energy balance. As a result, MaxEW can keep all sensors operating for as long as possible.

\section{Conclusion and Future Works}

Sensor networks should be able to achieve energy balance as well as energy efficiency. Most energy-aware routing algorithms are only concerned about energy efficiency. This paper has presented a performance measure, called Energy Welfare, to consider energy balance and efficiency of sensor networks simultaneously. Based on this metric, the proposal is for a Maximum Energy Welfare (MaxEW) routing algorithm. We demonstrate the superiority of this routing algorithm to Direct Communication, MTE, and SOR with a lifetime metric, generally accepted for evaluation of routing algorithms. Additionally, from the experimental results, the conclusion is that MaxEW is robust for several event generation functions.

To build the metric $E W$ and MaxEW algorithm, we here use the Atkinson welfare function and set the inequality aversion parameter to 2.5. Many alternative welfare functions are available in social science, and this inequality aversion parameter is tunable. In the future, we will apply alternate social welfare functions and different aversion parameters to enhance our results. Currently, we used three types of event generation function for evaluation of our routing algorithm. Future work will involve development of more diverse and detailed event generation functions.

In addition, we can consider a general multi-hop communication scenario where only a few sensors can communicate with the base station. In this scenario, the required transmission energy from a sensor to the base station can be calculated by the number of hop to the base station. As a future work, we will investigate how MaxEW works well in the general multi-hop scenario.

Acknowledgments. This work was supported in part by NSF under the grant NSFSST 0427840. Any opinions, findings, and conclusions or recommendations presented in this paper are those of the authors and do not necessarily reflect the views of the National Science Foundation.

\section{References}

1. D. Estrin, R. Govindan, J. Heidemann, and S. Kumar, Next century challenges: scalable coordination in sensor networks, Proceedings of the 5th annual ACM/IEEE international conference on Mobile computing and networking, Seattle, Washington, United States, 263270 (1999).

2. I. F. Akyildiz, W. Su, Y. Sankarasubramaniam, and E. Cayirci, A survey on Sensor Networks, IEEE Communications Magazine, 38(8), 102-114 (2002). 
3. D. Braginsky and D. Estin, Rumor Routing Algorithm for Sensor Networks, the proceedings of the first Workshop on Sensor Networks and Applications (WSNA), 22-31 (2002).

4. A. Rogers, E. David, and N. R. Jennings, Self-organized routing for wireless microsensor networks, IEEE Transactions on Systems, Man and Cybernetics, Part A, 35(3), 349-359 (2005).

5. Z. Butler and D. Rus, Event-based motion control for mobile-sensor networks, IEEE Pervasive Computing, 2(4), 34-42 (2003).

6. S. Lindsey, C. Raghavendra, and K. M. Sivalingam, Data Gathering Algorithms in Sensor Networks Using Energy Metrics, IEEE TRANSACTIONS ON PARALLEL AND DISTRIBUTED SYSTEMS, 13(9), 924-935 (2002).

7. W. R. Heinzelman, A. Chandrakasan, and H. Balakrishnan, Energy-Efficient Communication Protocol for Wireless Microsensor Networks, Proceedings of the 33rd Hawaii International Conference on System Sciences - 2000, (2000).

8. J. Chang and L. Tassiulas, Maximum Lifetime Routing in Wireless Sensor Networks, IEEE/ACM TRANSACTION ON NETWORKING, 12(4), 609-619 (2004).

9. J. Wanger and R. Cristescu, Power Control for Target Tracking in Sensor Networks, 2005 Conference on Information Sciences and Systems, The Johns Hopkins University, (2005).

10. R. Ramanathan and R. Rosales-Hain, Topology Control of Multihop Wireless Networks using Transmit Power Adjustment, INFOCOM 2000, 404-413 (2000).

11. H. Zhang and J. C. Hou, Maximizing $\alpha$-Lifetime for Wireless Sensor Networks, SenMetrics 2005, 70-77 (2005).

12. R. Wattenhofer, L. Li, P. Bahl, and Y. Wang, Distributed topology control for power efficient operation in multihop wireless ad hoc networks, INFORCOM 2001, Anchorage, AK, USA, 1388-1397 (2001).

13. M. Stemm and R. H. Katz, Measuring and reducing energy consumption of network interface in hand-held devices, IEICE Transactions on Communications, E80-B(8), 11251131 (1997).

14. S. R. Gandham, M. Dawande, R. Prakash, and V. S., Energy Efficient Schemes for Wireless Sensor Networks with Multiple Mobile Base Stations, Proceedings of IEEE Globecom 2003, 377-381 (2003).

15. A. B. Atkinson, On the measurement of inequality, Journal of Economics Theory, 2(3), 244-263 (1970).

16. D. A. Seiver, A note on the measurement of income inequality with interval data, Review of Income and Wealth, 25(2), 229-233 (1979).

17. J. G. Williamson, Strategic wage goods, prices, and inequality, American Economic Review, 67(2), 29-41 (1977).

18. A. K. Sen and J. E. Foster, On Economic Inequality, (Oxford: Clarendon Press, 1997). 